\title{
GeXP analyzer-based multiplex reverse- transcription PCR assay for the simultaneous detection and differentiation of eleven duck viruses
}

Yan-fang Zhang, Zhi-xun Xie ${ }^{*}$, Li-ji Xie, Xian-wen Deng, Zhi-qin Xie, Si-si Luo, Li Huang, Jiao-ling Huang and Ting-ting Zeng

\begin{abstract}
Background: Duck viral pathogens primarily include the avian influenza virus (AIV) subtypes $\mathrm{H} 5, \mathrm{H}$ 7, and $\mathrm{H}$; duck hepatitis virus (DHV); duck tembusu virus (DTMUV); egg drop syndrome virus (EDSV); duck enteritis virus (DEV); Newcastle disease virus (NDV); duck circovirus (DuCV); muscovy duck reovirus (MDRV); and muscovy duck parvovirus (MDPV). These pathogens cause great economic losses to China's duck breeding industry.

Result: A rapid, specific, sensitive and high-throughput GeXP-based multiplex PCR assay consisting of chimeric primer-based PCR amplification with fluorescent labeling and capillary electrophoresis separation was developed and optimized to simultaneously detect these eleven viral pathogens. Single and mixed pathogen cDNA/DNA templates were used to evaluate the specificity of the GeXP-multiplex assay. Corresponding specific DNA products were amplified from each pathogen. Other pathogens, including duck Escherichia coli, duck Salmonella, duck Staphylococcus aureus, Pasteurella multocida, infectious bronchitis virus, and Mycoplasma gallisepticum, did not result in amplification products. The detection limit of GeXP was $10^{3}$ copies when all twelve pre-mixed plasmids containing the target genes of eleven types of duck viruses were present. To further evaluate the reliability of GeXP, 150 clinical field samples were evaluated. Comparison with the results of conventional PCR methods for the field samples, the GeXP-multiplex PCR method was more sensitive and accurate.
\end{abstract}

Conclusion: This GeXP-based multiplex PCR method can be utilized for the rapid differential diagnosis of clinical samples as an effective tool to prevent and control duck viruses with similar clinical symptoms.

Keywords: Duck virus, Genome Lab Gene Expression Profiler (GeXP), Multiplex PCR, Separation identification

\section{Background}

According to the Food and Agriculture Organization (FAO) statistics, the 771 million ducks annually raised for meat purposes in China accounted for $65.73 \%$ of the world's stock in 2009 (FAO, 2009). There are eleven important viral pathogens that cause infections in ducks in China, including the avian influenza virus (AIV) subtypes H5, H7, H9; duck hepatitis virus (DHV); duck tembusu virus (DTMUV); egg drop syndrome virus

\footnotetext{
* Correspondence: xiezhixun@126.com
Department of Biotechnology, Guangxi Key Laboratory of Animal Vaccines

*Correspondence: xiezhixun@126.com
Department of Biotechnology, Guangxi Key Laboratory of Animal Vaccines and Diagnostics, Guangxi Veterinary Research Institute, 51 Youai North Road, Nanning 530001, China
}

(EDSV); duck enteritis virus (DEV); Newcastle disease virus (NDV); duck circovirus (DuCV); muscovy duck reovirus (MDRV); and muscovy duck parvovirus (MDPV) [1-4]. With the development of the commercial duck industry in China and in other parts of the world in recent years, the incidence of duck diseases has increased [5].

The increase in duck disease has become an important that restricts the growth and further development of the duck industry. Differential diagnosis of infectious duck diseases using traditional methods requires isolation of the pathogens and identification using serological techniques [6-9]. The accuracy of these methods is 
often affected by the freshness of the clinical material, contamination with bacteria and the length of time between the collection of the material and its analysis. Thus conventional methods of diagnosis are very tedious and time-consuming and are further complicated by the differential diagnosis of mixed infections $[10,11]$. Molecular typing methods for the rapid detection and identification of pathogens have been developed and are used currently. Although useful, most molecular methods are limited to the detection of a few pathogens in one reaction [12-16]. Therefore, a rapid, costeffective and high-throughput method for the detection and differentiation of viral pathogens in one test tube would be advantageous and would ensure prompt treatment and infection control.

The GenomeLab Gene Expression Profiler (GeXP) analyzer is a multiplex gene expression analysis platform developed by Beckman Coulter (Brea, CA, USA). GeXP assays have been successfully used for rapid identification in human medicine including assays to detect several inflammatory and cytokine gene targets in normal colon tissue as well as in colon polyps and tumors $[17,18]$, prostate cancer biomarker gene expression signatures in biological samples [19], pandemic influenza A H1N1 virus [20]; nine types of enteroviruses associated with hand, foot, and mouth disease [21]; and eleven human papillomaviruses in a rapid and sensitive manner [22]. Recently, a GeXP assay was used for the detection of nine avian respiratory disease agents [23]. The GeXP analyzer has a built-in software program that can be used to evaluate PCR products based on amplicon size. The software displays the differences between the various specific genes under investigation and compares the expected PCR product sizes to identify each product. The GeXP-multiplex PCR assay provide high sensitivity and specificity compared to other multiple-detection methods [24].

In this study, a GeXP analyzer-based multiplex RTPCR assay (GeXP-multiplex PCR) was developed to simultaneously detect eleven common duck viral diseases in China, including AIV-H5, AIV-H7, AIV-H9, DHV, DTMUV, EDSV, DEV, NDV, DuCV, MDRV, and MDPV. The diagnostic sensitivities and specificities of this assay were evaluated with 150 clinical specimens.

\section{Methods}

\section{Ethics statement}

This study was approved by the Institutional Animal Care and Use Committee (IACUC) of the Guangxi Veterinary Research Institute. Some specimens were collected from ducks that had died from disease. The other biological samples were briefly and gently collected from the larynx, trachea and cloaca of healthy ducks using sterilized cotton swabs without anesthesia after receiving the verbal permission of the owners' verbal permission. The sampled ducks were observed for $30 \mathrm{~min}$ after sampling and were then returned to their cages.

\section{Extraction of DNA/RNA from pathogens and sample preparation}

The avian pathogen reference strains, field isolates and other duck pathogens used in this study are described in Table 1. Viral RNA and DNA were extracted using the MiniBEST Viral RNA/DNA Extraction Kit Ver5.0 (Takara, Dalian, China) according to the manufacturer's protocol. The DNA and RNA samples were aliquoted and stored at $-30{ }^{\circ} \mathrm{C}$ until use.

\section{Primer design and plasmid preparation}

The GeXP-multiplex assay consists of twelve pairs of chimeric primers including one pair of AIV universal primers (AIV $M$ ) and eleven pairs of duck virus primers. These twelve pairs of duck virus primers were designed based on the sequences of the type A AIV $M$ gene; the $H A$ genes from the $\mathrm{H} 5$ and $\mathrm{H} 7$ and $\mathrm{H} 9$ subtypes of AIV; the 5'UTR region of the DHV gene; the DTMUV E gene; the DEV UL6 gene; the NDV $L$ gene; the EDSV Penton gene; the MDRV $S 1$ gene; the MDPV VP1 gene and the DuCV Red gene. All sequences were obtained from GenBank. The primers were designed by selecting the highly conserved regions using DNAstar software (DNASTAR Inc., Madison, WI, USA) and Primer Premier 5.0 software (Premier Biosoft International, Palo Alto, CA, USA). AIV universal primers were utilized to amplify all 16 subtypes. The $5^{\prime}$ end of the forward universal primer (Tag-F: AGGTGACACTATAGAATA) was labeled and purified with high-pressure liquid chromatography. All chimeric primers and the reverse universal primer (Tag-R: GTACGACTCACTATAGGGA) were synthesized and purified by polyacrylamide gel electrophoresis (BGI, China). The primer sequences, the size of the resulting amplicons, and the target regions are listed in Table 2.

Twelve specific genes from the eleven duck pathogens were amplified using the primers listed in Table 2. Plasmidencoding genes from DEV (AV1221), EDSV (GEV), MDPV (GX-5) and DuCV (GX1006) were prepared and quantified according to Xie $(26,27)$. Plasmids encoding genes from AIV (H5N1 AIV Re-1, H7N2 AIV Duck/HK/47/76, and H9N6/Duck/HK/147/77), DHV (AV2111), DTMUV (GX201301), NDV (GX1/00) and MDRV (NM1) were used to produce ssRNA via in vitro transcription using a High-Yield Transcription Kit (Thermo Scientific, USA). The copy numbers of the ssRNAs for AIV (H5N1 AIV Re-1, H7N2 AIV Duck/HK/47/76, H9N6/ Duck/HK/147/77), DHV (AV2111), DTMUV (GX201301), NDV (GX1/00) and MDRV (NM1) were calculated as described previously [23, 25]. 
Table 1 Sources of pathogens used and GeXP assay results

\begin{tabular}{|c|c|c|c|c|c|c|c|c|c|c|c|c|c|c|}
\hline \multirow[t]{2}{*}{ Pathogen/field samples } & \multirow{2}{*}{$\begin{array}{l}\text { Number of } \\
\text { samples }\end{array}$} & \multirow[t]{2}{*}{ Source } & \multicolumn{12}{|c|}{ Results } \\
\hline & & & $\begin{array}{l}\text { AlV- } \\
\mathrm{M}\end{array}$ & $\begin{array}{l}\text { AlV- } \\
\mathrm{H} 5\end{array}$ & $\begin{array}{l}\text { AlV- } \\
\mathrm{H} 7\end{array}$ & $\begin{array}{l}\text { AlV- } \\
\mathrm{H} 9\end{array}$ & $\mathrm{DHV}$ & DEV & DTMUV & NDV & EDSV & MDRV & MDPV & DuCV \\
\hline \multicolumn{15}{|l|}{ Reference sample } \\
\hline${ }^{\mathrm{a}} \mathrm{CDNA}$ of H5N3 AIV Duck/HK 313/78 & 1 & $\mathrm{HKU}$ & + & + & - & - & - & - & - & - & - & - & - & - \\
\hline aH7N2 AIV Duck/HK 47/76 & 1 & $\mathrm{HKU}$ & + & - & + & - & - & - & - & - & - & - & - & - \\
\hline a'H9N6 AlV Duck/HK/147/77 & 1 & $\mathrm{HKU}$ & + & - & - & + & - & - & - & - & - & - & - & - \\
\hline bDHV (AV2111) & 1 & CIVDC & - & - & - & - & + & - & - & - & - & - & - & - \\
\hline${ }^{\circ} \mathrm{DEV}(\mathrm{AV} 1221)$ & 1 & CIVDC & - & - & - & - & - & + & - & - & - & - & - & - \\
\hline dDTMUV (GX201301,GX201302) & 2 & GVRI & - & - & - & - & - & - & + & - & - & - & - & - \\
\hline eNDV (GX1/00,GX6/02) & 2 & GVRI & - & - & - & - & - & - & - & + & - & - & - & - \\
\hline fEDSV (GEV) & 1 & GVRI & - & - & - & - & - & - & - & - & + & - & - & - \\
\hline${ }^{9} \mathrm{MDRV}(\mathrm{NM1}, \mathrm{NM} 2)$ & 2 & GVRI & - & - & - & - & - & - & - & - & - & + & - & - \\
\hline${ }^{h} M D P V(G X-5, G X-6)$ & 2 & GVRI & - & - & - & - & - & - & - & - & - & - & + & \\
\hline 'DuCV (GX1006,GX1008) & 2 & GVRI & - & - & - & - & - & - & - & - & - & - & - & + \\
\hline \multicolumn{15}{|l|}{ Other pathogens } \\
\hline H1N3 AIVDuck/HK/717/79-d1 & 1 & $\mathrm{HKU}$ & + & - & - & - & - & - & - & - & - & - & - & - \\
\hline H1N1 AIV Human/NJ/8/76 & 1 & $\mathrm{HKU}$ & + & - & - & - & - & - & - & - & - & - & - & - \\
\hline H2N3 AlV Duck/HK/77/76 & 1 & $\mathrm{HKU}$ & + & - & - & - & - & - & - & - & - & - & - & - \\
\hline H3N6 AIV Duck/HK/526/79/2B & 1 & $\mathrm{HKU}$ & + & - & - & - & - & - & - & - & - & - & - & - \\
\hline H4N5 AIV Duck/HK/668/79 & 1 & $\mathrm{HKU}$ & + & - & - & - & - & - & - & - & - & - & - & - \\
\hline H6N8 AlV Duck/HK/531/79 & 1 & $\mathrm{HKU}$ & + & - & - & - & - & - & - & - & - & - & - & - \\
\hline H8N4AIV Turkey/ont/6118/68 & 1 & $\mathrm{HKU}$ & + & - & - & - & - & - & - & - & - & - & - & - \\
\hline H10N3 AIV Duck/HK/876/80 & 1 & $\mathrm{HKU}$ & + & - & - & - & - & - & - & - & - & - & - & - \\
\hline H11N3 AIV Duck/HK/661/79 & 1 & $\mathrm{HKU}$ & + & - & - & - & - & - & - & - & - & - & - & - \\
\hline H12N5 AIV Duck/HK/862/80 & 1 & $\mathrm{HKU}$ & + & - & - & - & - & - & - & - & - & - & - & - \\
\hline H6N8 AIV Duck/HK/531/79 & 1 & $\mathrm{HKU}$ & + & - & - & - & - & - & - & - & - & - & - & - \\
\hline duck Escherichia coli & 1 & GVRI & - & - & - & - & - & - & - & - & - & - & - & - \\
\hline duck Salmonella & 1 & GVRI & - & - & - & - & - & - & - & - & - & - & - & - \\
\hline duck Staphylococcus aureus & 1 & GVRI & - & - & - & - & - & - & - & - & - & - & - & - \\
\hline Pasteurella multocida & 1 & GVRI & - & - & - & - & - & - & - & - & - & - & - & - \\
\hline Infectious bronchitis virus & 1 & GVRI & - & - & - & - & - & - & - & - & - & - & - & - \\
\hline Mycoplasma gallisepticum & 1 & GVRI & - & - & - & - & - & - & - & - & - & - & - & - \\
\hline \multicolumn{15}{|l|}{ Sample mixture } \\
\hline AlV-H5 + AlV-H7 + AlV-H9 & 1 & GVRI & + & + & + & + & - & - & - & - & - & - & - & - \\
\hline AIV-H5 + DEV + DTMUV + NDV + EDSV & 1 & GVRI & + & + & - & - & - & + & + & + & + & - & - & - \\
\hline $\begin{array}{l}\text { AlV-H5 + AlV-H7 + AIV-H9 + DHV + DEV + } \\
\text { DTMUV + NDV + EDSV+ MDRV + MDPV+ DuCV }\end{array}$ & 1 & GVRI & + & + & + & + & + & + & + & + & + & + & + & + \\
\hline
\end{tabular}

HVRI Harbin Veterinary Research Institute, China, HKU The University of HongKong, China, GVRI Guangxi Veterinary Research Institute, China, CIVDC China Institute of Veterinary Drug Control, China, PU University of Pennsylvania

aeferences [23]

${ }^{\mathrm{b}}$ GenBank accession no. : EF442073.1

'GenBank accession no. : EU315247

${ }^{\mathrm{d} G e n B a n k}$ accession no. : KJ700462.1

${ }^{\mathrm{e}}$ GenBank accession no. : JX193083.1

fReferences [35]

${ }^{9}$ References [35]

${ }^{\mathrm{h}}$ GenBank accession no. : KM093740.1

'GenBank accession no. : JX241046.1 
Table 2 Primers used in this study

\begin{tabular}{|c|c|c|c|c|}
\hline Virus & Forward primer sequence $\left(5^{\prime}-3^{\prime}\right)$ & Reverse primer sequence $\left(5^{\prime}-3^{\prime}\right)$ & Amplicon size (bp) & Target region \\
\hline AIV-M & AGGTGACACTATAGAATACAGAAACGGATGGGAGTGC & GTACGACTCACTATAGGGATATCAAGTGCAAGATCCCAATGAT & 122 & $M$ \\
\hline AIV-H5 & AGGTGACACTATAGAATACTTCAGGCATCAAAATGCACA & GTACGACTCACTATAGGGATAGTTTGTTCATTTCTGAGTCGGTC & 285 & $H A$ \\
\hline AIV-H7 & AGGTGACACTATAGAATAAATGGGGCHTTCATAGCTCC & GTACGACTCACTATAGGGATGATAGCARTCRCCTTCACAA & 144 & $H A$ \\
\hline AIV-H9 & AGGTGACACTATAGAATAACAACAAGTGTGACAACAGAAGA & GTACGACTCACTATAGGGATCTTCCGTGGCTCTCTCC & 237 & $H A$ \\
\hline DHV & AGGTGACACTATAGAATATCTTCGTTGTGAAACGGATTACC & GTACGACTCACTATAGGGATGCCTGGACAGATDTGTGCCTACT & 133 & $5^{\prime}$ UTR \\
\hline DTMUV & AGGTGACACTATAGAATAATGGACAGGGTCATCAGCGG & GTACGACTCACTATAGGGAGAATRGCTCCYGCCAATGCT & 176 & $E$ \\
\hline EDSV & AGGTGACACTATAGAATAAATCGGCAACTCAAGACATC & GTACGACTCACTATAGGGACCCATTCATAAACAGGATTC & 208 & Penton \\
\hline DEV & AGGTGACACTATAGAATAGGGAGGAGCAAACAAAGA & GTACGACTCACTATAGGGAATCGCAAATTCCATCACATA & 150 & UL6 \\
\hline NDV & AGGTGACACTATAGAATAGTRGCAGCAAGRACAAGG & GTACGACTCACTATAGGGACATATCYGCATACATCAA & 196 & $L$ \\
\hline DuCV & AGGTGACACTATAGAATATGCKCCAAAGAGTCGACATA & GTACGACTCACTATAGGGACAAAYGCATAACGGCTCTTTCC & 300 & Red \\
\hline MDRV & AGGTGACACTATAGAATACAGTTGAGCCGGAYGGTAATT & GTACGACTCACTATAGGGAACTCGGTTGGTGTTAGTVGCVTAGAA & 219 & S1 \\
\hline MDPV & AGGTGACACTATAGAATACTTTCAGGCTACATCTTCAA & GTACGACTCACTATAGGGAAATTCTCTTTTCACCCATCC & 253 & VP1 \\
\hline
\end{tabular}

Universal tag sequences Tag-F: AGGTGACACTATAGAATA Tag-R: GTACGACTCACTATAGGGA

Universal tag sequences are underlined. Bold type indicates degenerate sites

Abbreviations: $M$ A or $\mathrm{C}, R \mathrm{~A}$ or $\mathrm{G}, W \mathrm{~A}$ or $\mathrm{T}, \mathrm{S} \mathrm{G}$ or $\mathrm{C}, Y \mathrm{C}$ or T, $K \mathrm{G}$ or T, V A, G, or C, H A, C, or T, D A, G, or T; B G, C, or T 


\section{Setup of the GeXP assay and the reaction procedure}

The reaction system was created using the GeXP Start-up Kit in a total volume of $20 \mu \mathrm{l}$ containing $4 \mu \mathrm{l}$ of Genome LabTM GeXP Start Kit $5 \times$ PCR Buffer, $4 \mu \mathrm{l}$ of $\mathrm{MgCl}_{2}$ $(25 \mu \mathrm{M}), 2 \mu \mathrm{l}$ of mixed primers (containing 20-100 nmol/l of 12 pairs of gene-specific chimeric primers), $1.4 \mu \mathrm{l}$ of JumpStart Taq DNA polymerase, and $0.5 \mathrm{pg}-0.5 \mathrm{ng}$ of cDNA or DNA template. Nuclease-free water was then added to the PCR reaction to achieve a final volume of $20 \mu \mathrm{l}$. Three optimized PCR amplification steps were performed according to the temperature-switch PCR (TSP) strategy [22]: step 1 was carried out with gene-specific sequences of chimeric forward and reverse primers (10 cycles of $30 \mathrm{~s}$ at $95^{\circ} \mathrm{C}, 30 \mathrm{~s}$ at $55^{\circ} \mathrm{C}$, and $30 \mathrm{~s}$ at $72{ }^{\circ} \mathrm{C}$ ); step 2 was carried out predominantly with chimeric forward and reverse primers $\left(10\right.$ cycles of $30 \mathrm{~s}$ at $95{ }^{\circ} \mathrm{C}, 30 \mathrm{~s}$ at $63{ }^{\circ} \mathrm{C}$, and $30 \mathrm{~s}$ at $72{ }^{\circ} \mathrm{C}$ ); and step 3 was carried out predominantly with universal forward and reverse primers $\left(20\right.$ cycles of $30 \mathrm{~s}$ at $95{ }^{\circ} \mathrm{C}, 30 \mathrm{~s}$ at $50{ }^{\circ} \mathrm{C}$, and $30 \mathrm{~s}$ at $72{ }^{\circ} \mathrm{C}$ ). The reactions were held at $4{ }^{\circ} \mathrm{C}$ after the amplification cycles.

\section{Separation by capillary electrophoresis and fragment analysis}

After amplification, $1 \mu \mathrm{l}$ of the PCR product was added to $20 \mu \mathrm{l}$ of sample loading solution along with $0.16 \mu \mathrm{l}$ of DNA Size Standard-400 (Genome Lab GeXP Start Kit Beckman Coulter), following protocols described previously [19]. After amplified DNA amplicons were separated, the data were imported into the analysis module of ExpressProfiler software as a tab-delimited file for subsequent analyses.

\section{Evaluating the specificity of the GeXP assay}

cDNA and DNA templates were used for the GeXPmono PCR assay and the GeXP-multiplex PCR assay. The GeXP-mono PCR assay was performed using a single template along with each pair of chimeric primers to determine the size of the amplification products for each target gene. The specificity of the GeXP-multiplex PCR assay was tested for each individual viral target gene, and the assay was performed using a single template. The mono-GeXP PCR assay and GeXP-multiplex PCR assay were developed using the reaction system and procedure described in Material and Methods Section 2.3.

\section{Evaluating the accuracy of the GeXP assay}

The reaction system and procedures described above were used for the validation of GeXP-multiplex PCR for the detection of the twelve viruses. cDNA/DNA hybrids of all twelve duck viral disease agents were used as templates along with a primer mixture $(0.2 \mu \mathrm{l})$. PCR product separation and detection were performed on a Genome Lab GeXP Genetic Analysis System (Beckman
Coulter) via capillary electrophoresis, following the protocols described previously in Materials and Methods 2.3.

After the amplified fragments were separated, the peaks were initially analyzed using the fragment analysis module of the GeXP system software and matched to the appropriate genes. The peak height for each gene was reported in the electropherogram.

To emulate mixed infections, we randomly chose duck pathogens and extracted their DNA and RNA. RNA viruses were transcribed into cDNA as described above. DNA and cDNA were mixed together in equal concentrations to serve as templates for the optimized GeXPmultiplex PCR assay. To simulate the detection of a clinically mixed infection by GeXP, random cDNAs from eleven viruses or DNA samples from three to five viruses were mixed, and two groups were established. Group 1 consisted of a mixture of cDNAs from three avian influenza viruses (AIV-H5, AIV-H7 and AIV-H9) per template, while group 2 consisted of cDNA/DNA mixtures of DEV, DTMUV, NDV, EDSV and AIV-H5 viruses per template. A mixture of primers $(0.2 \mu \mathrm{l})$ was added, and the remainder of the procedure was performed as described in Materials and Methods.

\section{Evaluating the sensitivity of the GeXP assay}

The sensitivity of the GeXP-multiplex assay for each type of duck virus was examined using serial 10-fold dilutions of the plasmids (DEV, EDSV, MDPV, and $\mathrm{DuCV}$ ) and ssRNAs obtained via in vitro transcription (AIV-5, AIV-7, AIV-9, DHV, DTMUV, NDV, and MDRV), either separately or with the twelve samples mixed together $\left(10^{6}\right.$ to $10^{1}$ copies/ $\left.\mu \mathrm{l}\right)$. The assays were performed in triplicate. Plasmid or ssRNA mixtures were used to test the detection limit when all twelve duck virus genotypes were present. PCR was performed using the same experimental conditions described above for the GeXP-multiplex PCR assay, and the detection limit of the GeXP-PCR was determined based on the most dilute template that yielded a positive result. After amplification, $2 \mu \mathrm{l}$ of each Cy5-labeled PCR product was separated via GeXP capillary electrophoresis and detected by fluorescence spectrophotometry.

\section{Interference assay}

Because the presence of other templates in high quantities could alter the efficiency of GeXP-multiplex PCR amplification, different amounts of the templates $\left(10^{3}\right.$ to $10^{7}$ copies) were selected at random, mixed and tested in the GeXP-multiplex PCR assay. The results were compared with those of the single-template GeXPmultiplex PCR assay. 
Evaluation of the GeXP-PCR assay using clinical samples A total of 150 archived clinical specimens were collected from ducks in Guangxi, China, between July, 2012 and November 2013. RNA and DNA from the 150 specimens were then extracted using the MiniBEST Viral RNA/DNA Extraction Kit (Takara, Dalian, China) and a TIANamp Bacteria DNA Kit (Tiangen, Beijing, China). Genomic RNA from all samples was transcribed into cDNA as described above, and cDNA samples were analyzed using the optimized GeXP-multiplex PCR assay in addition to conventional simplex PCR methods with same primers as the GeXP assay. The obtained positive samples were send to a company (BGI, China) for sequencing.

\section{Results}

\section{Specificity of the GeXP-multiplex PCR assay}

The DNA and cDNA from eleven duck pathogens were used as templates to evaluate the specificity of each pair of gene-specific primers. In mono-GeXP PCR assays, the AIV universal primers that we designed could amplify the target $M$ genes of all AIV serotypes; however, each pair of pathogen-specific primers amplified only the corresponding genes from the targeted pathogens without cross-amplification. The expected amplification peaks were observed in the GeXP-multiplex PCR assay (Table 1). No specific amplification peaks were observed when duck Escherichia coli, duck Salmonella, duck
Staphylococcus aureus, Pasteurella multocida, infectious bronchitis virus, or Mycoplasma gallisepticum were tested (Table 1).

The GeXP-multiplex PCR assay used twelve pairs of primers to detect eleven duck viruses, and specific amplification peaks were observed (Fig. 1). Specific amplification peaks corresponding to the $M$ gene of AIV were observed upon testing H1N3, H1N1, H2N3, H3N6, H4N5, H6N8, H8N4, H10N3, H11N3, H12N5 and H13N5.

\section{Sensitivity of the GeXP assay}

The GeXP-PCR assay was capable of detecting as few as 10-100 copies of each of the twelve recombinant plasmids of duck viruses (data not shown) and as few as $10^{3}$ copies when all of the twelve pre-mixed duck virus targets were present in a mixture (Fig. 2). Typically, a reaction is considered positive when the A.U. value is over 2000 by default [22], and 10 duck viruses were detected at a concentration of $10^{2}$ copies per reaction ( $\mathrm{DuCV}$ being the exception). The reactions were repeated three times at each template concentration, and similar results were obtained.

\section{Artificial mixture}

The cDNA and DNA from the duck pathogens were mixed together to test the ability of the GeXP-multiplex PCR assay to differentiate among them, and the
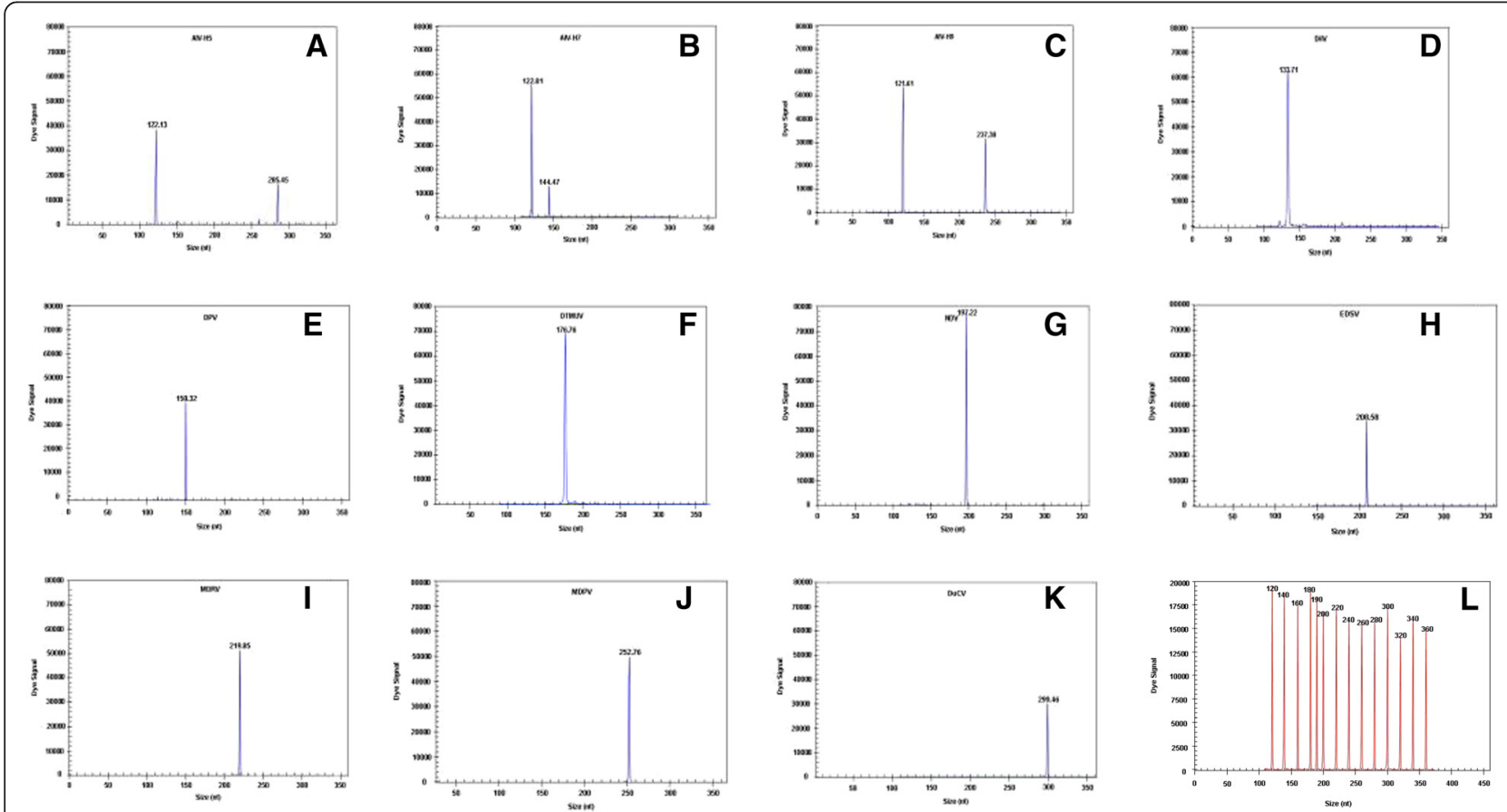

Fig. 1 Specificity analyses of the GeXP-PCR assay. The Y-axis indicates the dye signal in A.U., and the X-axis indicates the actual PCR product size. a-k show the results of the amplification of AIV-H5, AIV-H7, AIV-H9, DHV, DEV, DTMUV, NDV, EDSV, MDRV, MDPV, and DuCV, respectively. Nuclease-free water was used as the negative control (I). The red peaks indicate the DNA size standard 

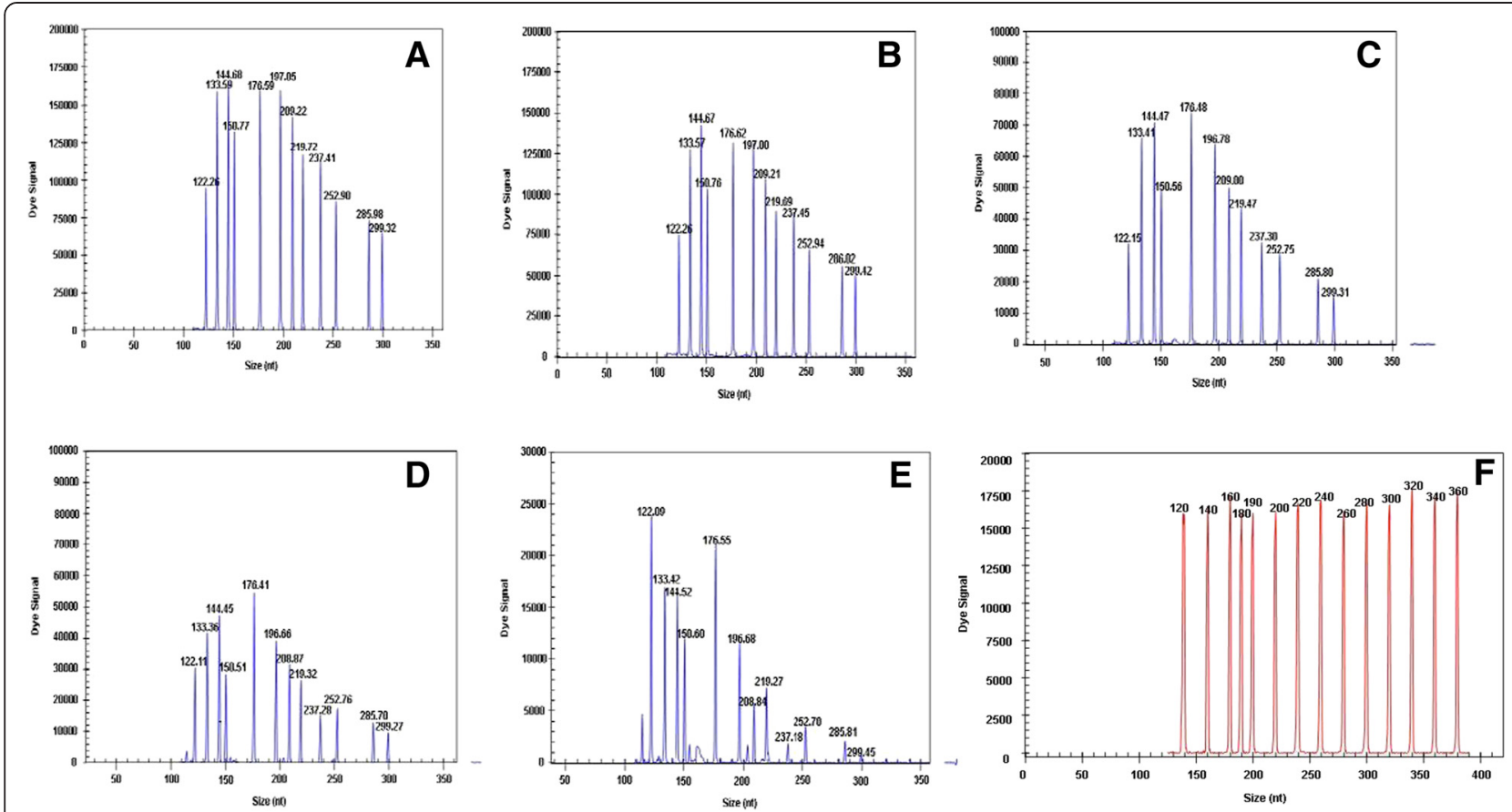

Fig. 2 Sensitivity of the GeXP-PCR assay. Serial 10-fold dilutions of plasmids containing the 12 duck virus types were prepared and amplified in the GeXP-PCR assay using equal amounts of template: $10^{6}(\mathbf{a}), 10^{5}(\mathbf{b}), 10^{4}(\mathbf{c}), 10^{3}(\mathbf{d})$ and $10^{2}(\mathbf{e})$ copies per reaction in the GeXP-PCR assay. The viral targets from left to right are as follow: AIV-M, DHV, AIV-H7, DEV, DTMUV, NDV, EDSV, MDRV, AIV-H9, MDPV, AIV-H5, and DuCV. Nuclease-free water was used as the negative control (f)

appropriate specific amplification peaks and universal amplification peaks were observed (Table 1; Fig. 3a, b). When AIV-H5, AIV-H7and AIV-H9 were mixed together and used in the GeXP-multiplex PCR assay, 3 specific amplification peaks (AIV-H7, 144.92 bp; AIVH9, $237.21 \mathrm{bp}$; AIV-H5, $285.73 \mathrm{bp)}$ and one universal amplification peak (AIV $M$ gene, $122.14 \mathrm{bp}$ ) were observed (Fig. 3a). When AIV-H5, DEV, DTMUV, NDV and EDSV were mixed together and used in the GeXPmultiplex PCR assay, there were six specific amplification peaks (Fig. 3b). When the cDNA and DNA of all eleven pathogens were mixed and used in the GeXPmultiplex PCR assay, eleven specific amplification peaks were observed (Table 1; Fig. 3c).

\section{Interference assay}

Three specific amplification peaks were observed when three different templates (one template with $10^{3}$ copies and two templates with $10^{7}$ copies) were tested using the GeXP-multiplex PCR assay. Additionally, the peak value of a single template was similar to that of a mixed template. For example, three specific amplification peaks were observed when three different templates $\left(10^{3}\right.$ copies of DuCV and $10^{7}$ copies of AIV-M and AIV-H9) were tested using the GeXP-multiplex PCR assay (Fig. 4), and the peak values for AIV-M, AIV-H9 and for DuCV were the same regardless of whether a single template (AIV-M and AIVH9 or DuCV) or a mixed template (AIV-M + AIV-H9 + $\mathrm{DuCV}$ ) was utilized. The results of these experiments
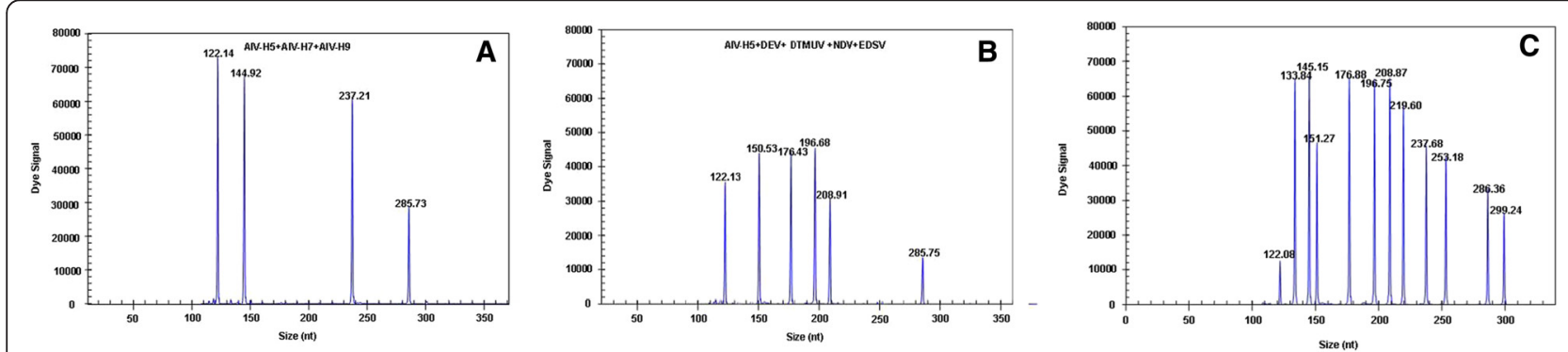

Fig. 3 GeXP-multiplex PCR detection of mixed pathogen templates. The GeXP-multiplex assay was carried out with mixed templates and mixed primers for AIV-H5, AIV-H7 and AIV-H9 (a); AIV-H5, DEV, DTUMV, NDV and EDSV (b); or all eleven viruses (c) 


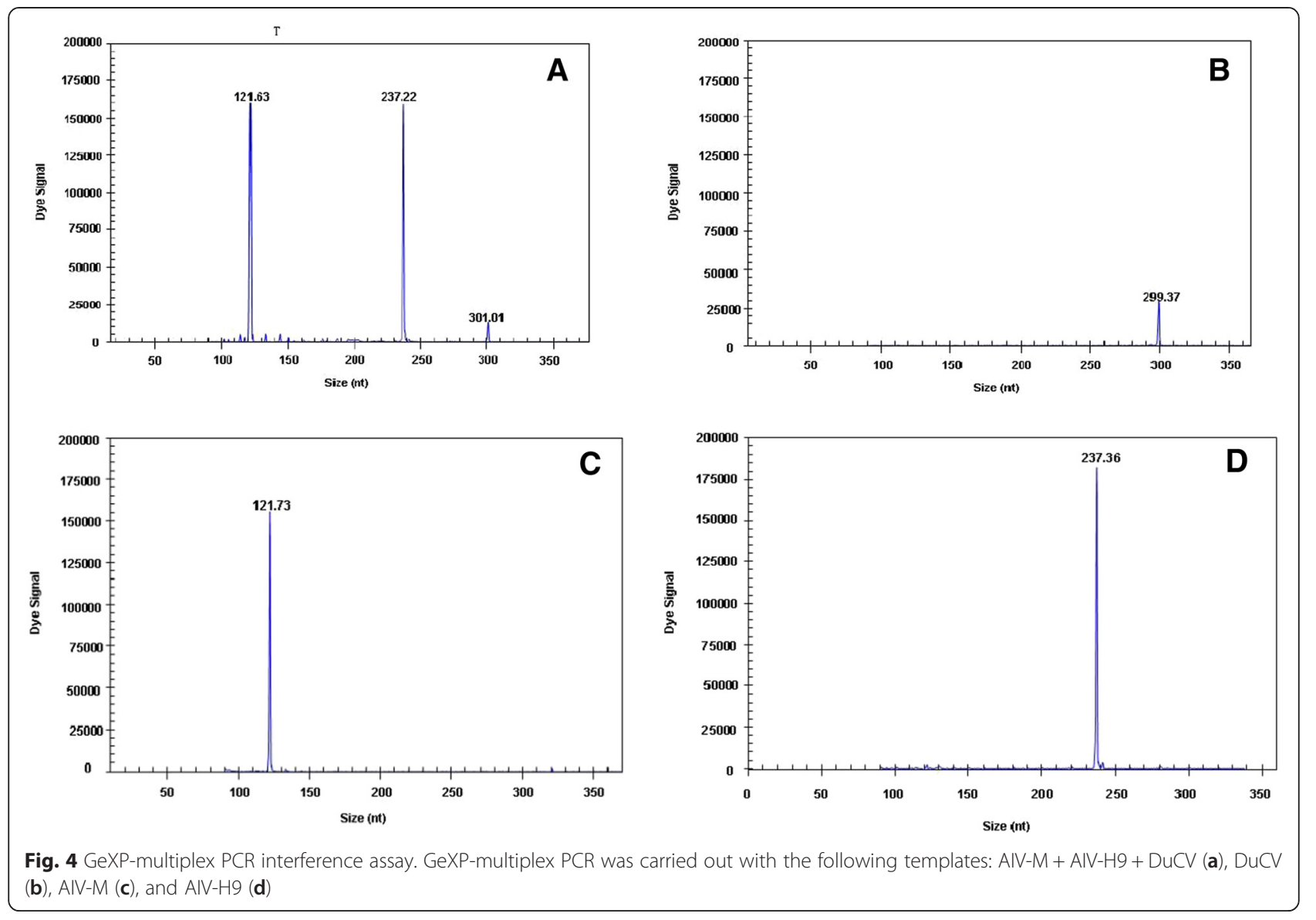

showed that mixed infections can be detected by GeXPmultiplex PCR with only minimal interference.

\section{Assay of field samples using GeXP-multiplex PCR}

A total of 150 clinical samples were assayed using the optimized GeXP-multiplex PCR method in addition to the conventional simplex PCR method. The positive and negative results obtained with the different methods are shown in Table 3. The degree of consistency between and the GeXP assay and conventional PCR methods is shown in Table 4. The kappa values as a measure of agreement for the GeXP assay and conventional PCR methods were as follow: $\mathrm{K}=0.908, \mathrm{~K}>0 ; \mathrm{u}=28.375$, $\mathrm{u}>1.96, P<0.05$. The two experimental results appear to have a high degree of consistency. All positive specimens in the GeXP assay and conventional methods were identified via sequencing (BGI, China) to be true positive samples.

\section{Discussion}

AIV-H5, AIV-H7, AIV-H9, DHV, DEV, DTMUV, NDV, EDSV, MDRV, MDPV, and DuCV are the eleven types of viruses that cause infectious diseases in duck. In this study, we developed a GeXP method for the simultaneous detection of these eleven duck viruses. Twelve pairs of specific primers were designed according to the conserved sequences of the genes from each pathogen; these sequences were obtained from GenBank. The GeXP genetic analysis system is a multitarget, high-throughput detection platform, and its application in the differential detection of nine avian respiratory pathogens was reported recently by our laboratory $[23,26]$.

The amplification specificity and accuracy of each primer set in GeXP-multiplex PCR was verified by the inspection of samples containing a single virus or a mixed population and positive clinical samples (including mixed infections, single infections and infections with more than two types of pathogen) were used to further verify the method. The evaluation of sensitivity using recombinant plasmids for each duck virus revealed that all twelve of the hybrid templates were detected simultaneously when they were present at a concentration of $10^{3}$ copies per reaction, indicating the high sensitivity of the GeXP-PCR assay. One hundred and fifty specimens were analyzed by the GeXP-PCR assay ( 89 were positive) and conventional PCR methods (81 were positive). Additionally, 8 clinical samples that were negative based on conventional single PCR but positive in the GeXP assay 
Table 3 Analysis of clinical samples using the GeXP assay and conventional PCR methods

\begin{tabular}{|c|c|c|c|c|}
\hline \multirow[t]{2}{*}{ Clinical samples } & \multicolumn{2}{|c|}{ No. of GeXP assay results } & \multicolumn{2}{|c|}{ No. of conventional PCR method results } \\
\hline & Positive & Negative & Positive & Negative \\
\hline H9 subtypes of AIV & 20 & 130 & 18 & 132 \\
\hline $\mathrm{DHV}$ & 5 & 145 & 5 & 145 \\
\hline DEV & 1 & 149 & 1 & 149 \\
\hline DTMUV & 5 & 145 & 5 & 145 \\
\hline NDV & 10 & 140 & 9 & 141 \\
\hline EDSV & 3 & 142 & 3 & 147 \\
\hline MDRV & 1 & 149 & 1 & 149 \\
\hline MDPV & 4 & 146 & 4 & 146 \\
\hline DuCV & 40 & 110 & 35 & 115 \\
\hline Total & 89 & 61 & 81 & 69 \\
\hline
\end{tabular}

were confirmed later by sequencing to be true positives (data not shown). Compared with the results of conventional PCR method, the GeXP-multiplex PCR method was more sensitive and accurate.

In recent years, multiplex PCR and multiplex fluorescence real-time quantitative PCR techniques have been widely used for the detection of mixed infections with multiple pathogens [27]. Multiple PCR involves the use of several primer pairs in one reaction system at the same time. Thus competition occurs during amplification as the primer pairs interfere with each other. The probability of forming complex primer dimers increases, and the sensitivity decrease. Additionally, multiplex PCR products are typically observed by agarose gel electrophoresis, which is not an optimal method for distinguishing bands that are less than $100 \mathrm{bp}$ in length. Furthermore, the probes used in multiplex fluorescence real-time quantitative PCR are conjugated to fluorophores that emit light at different wavelengths. General PCR methods, such as conventional multiplex PCR and multiplex real-time PCR [28-32], can only detect 2 to 6 types of pathogens; thus, these methods are not ideal for the rapid high-throughput detection of gene expression or analysis of multiple pathogens. Quantitative GeXP expression analysis combines multiplex PCR and capillary electrophoresis, employing fluorescently labeled universal primers and specific primer combinations to trigger multiple amplifications. Using this technique, multiple genes can be amplified using 1

Table 4 Comparison of clinical samples using the GeXP assay and conventional PCR methods

\begin{tabular}{llll}
\hline $\begin{array}{l}\text { GeXP assay } \\
\text { results }\end{array}$ & \multicolumn{2}{l}{ Conventional PCR methods results } & Total \\
\cline { 2 - 3 } & + & - & \\
\hline+ & 81 & 8 & 89 \\
- & 0 & 61 & 61 \\
Total & 81 & 69 & 150 \\
\hline
\end{tabular}

pair of universal primers. The amplification efficiency of each template is consistent, and the amplification efficiency of each primer pair is not affected. Thus, highthroughput detection and identification of multiple pathogens can be achieved using this method.

Two other distinct advantages of this GeXP-multiplex PCR method are the short assay time and the low cost $[33,34]$. The entire reaction can be completed in one tube within $2.5 \mathrm{~h}$ followed by capillary electrophoresis separation. In addition, two 96-well plates can be placed in parallel in a GeXP analyzer at the same time to further increase the throughput of this method.

The findings described here may lead to increased utilization of these gene-based tests for the routine diagnosis of viral infection. In addition, some of the viruses we detected in the assay may not be pathogenic in animals or could be live virus vaccine strains found in the animal herds. However, the data may still be valuable for epidemiological studies. To make a definite diagnosis, we must consider the clinical symptoms and whether live vaccine was recently injected. Furthermore, we have found that the GeXP analyzer is an efficient and easy-to-use tool; thus it may be a useful new technology for the identification of new biomarkers for diagnosing viral diseases in ducks.

\section{Conclusions}

This study has demonstrated that the GeXP-multiplex PCR assay is a rapid method with high sensitivity and specificity for the identification of three very important avian influenza subtypes (H5, H7, H9) in addition to eight other duck pathogens. This method may therefore be adopted for the molecular epidemiologic surveillance of these duck pathogens.

\footnotetext{
Abbreviations

AIV: Avian influenza virus; DHV: Duck hepatitis virus; DTMUV: Duck tembusu virus; EDSV: Egg drop syndrome virus; DEV: Duck enteritis virus; NDV: NEWCASTLE disease virus; DuCV: Duck circovirus; MDRV: Muscovy duck reovirus; MDPV: Muscovy duck parvovirus; GeXP: Genome Lab Gene Expression Profiler; IACUC: Institutional Animal Care and Use Committee; TSP: Temperature-switch PCR.
} 


\section{Competing interests}

The authors declare that they have no competing interests. A Chinese patent (application number: 201410037453.1) has been previously filed for the combination of all of the primers specific to the 11 duck viruses listed in this study and the experimental parameters used for the RT-PCR assay. This technology is available for research only.

\section{Authors' contributions}

Zhi-xun Xie designed and coordinated the study. Yan-fang Zhang designed the primers and optimized the conditions of the GeXP assay. Li-ji Xie was responsible for providing the clinical samples and leading the evaluation of the GeXP results with Xian-wen Deng. Zhi-qin Xie, Si-si Luo, Li Huang offered a great deal of help in the supplementing different clinical samples as well as sharing previous experimental data. Yan-fang Zhang performed the data analysis and wrote the manuscript. Jiao-ling Huang and Ting-ting Zeng reviewed and edited the manuscript. All authors read and approved the final version of the manuscript.

\section{Acknowledgements}

This work was supported by the Guangxi Science and Technology Bureau (1222003-2-4 and 10100014-5) and by the Guangxi Government Senior Scientist Foundation (2011B020) (Guangxi, China).

\section{Received: 29 April 2015 Accepted: 23 October 2015}

\section{Published online: 30 October 2015}

\section{References}

1. Zhang D. The current epidemic status of duck disease and its prevention and control strategies. China Poultry. 2012;34:38-40.

2. Butterfield WK, Ata FA, Dardiri AH. Duck plague virus distribution in embryonating chicken and duck eggs. Avian Dis. 1969;13(1):198-202.

3. Liu P, Lu H, Li S, Wu Y, Gao GF, Su J. Duck egg drop syndrome virus: an emerging Tembusu-related flavivirus in China. Sci China Life Sci. 2013;56(8):701-10

4. $\quad$ Ren L, Li J, Bi Y, Chen C, Zhang D, Liu W. Overview on duck virus hepatitis A. Sheng Wu Gong Cheng Xue Bao. 2012;28(7):789-99.

5. Hou S. The waterfowl industry faced with the challenge in China. China Poultry. 2013;35(10):36-7.

6. Plummer PJ, Alefantis T, Kaplan S, O'Connell P, Shawky S, Schat KA Detection of duck enteritis virus by polymerase chain reaction. Avian Dis. 1998;42(3):554-64.

7. Hotta K, Takakuwa H, Yabuta T, Ung TTH, Usui T, Nguyen HLK, et al. Antibody survey on avian influenza viruses using egg yolks of ducks in Hanoi between 2010 and 2012. Vet Microbiol. 2013;166(1-2):179-83.

8. Schmitz A, Le Bras MO, Guillemoto C, Pierre I, Rose N, Bougeard S, et al. Evaluation of a commercial ELISA for $\mathrm{H} 5$ low pathogenic avian influenza virus antibody detection in duck sera using Bayesian methods. J Virol Methods. 2013;193(1):197-204.

9. Wang G, Qu Y, Wang F, Hu D, Liu L, Li N, et al. The comprehensive diagnosis and prevention of duck plague in northwest Shandong province of China. Poult Sci. 2013;92(11):2892-8.

10. Mason RA, Tauraso NM, Ginn RK. Growth of duck hepatitis virus in chicken embryos and in cell cultures derived from infected embryos. Avian Dis. 1972;16(5):6

11. Zhang Y, Li Y, Liu M, Zhang D, Guo D, Liu C, et al. Development and evaluation of a VP3-ELISA for the detection of goose and Muscovy duck parvovirus antibodies. J Virol Methods. 2010;163(2):405-9.

12. Ji J, Xie QM, Chen CY, Bai SW, Zou LS, Zuo KJ, et al. Molecular detection of Muscovy duck parvovirus by loop-mediated isothermal amplification assay. Poult Sci. 2010;89(3):477-83.

13. Liu SN, Zhang XX, Zou JF, Xie ZJ, Zhu YL, Zhao Q, et al. Development of an indirect ELISA for the detection of duck circovirus infection in duck flocks. Vet Microbiol. 2010;145(1-2):41-6.

14. Wozniakowski G, Samorek-Salamonowicz E, Kozdrun W. Quantitative analysis of waterfowl parvoviruses in geese and Muscovy ducks by realtime polymerase chain reaction: correlation between age, clinica symptoms and DNA copy number of waterfowl parvoviruses. BMC Vet Res. 2012;8:29.

15. Xie Z, Xie L, Xu Z, Liu J, Pang Y, Deng X, et al. Identification of a Genotype IX Newcastle Disease Virus in a Guangxi White Duck. Genome Announc. 2013;1(5):e00836.
16. Xie L, Xie Z, Zhao G, Liu J, Pang Y, Deng X, et al. A loop-mediated isothermal amplification assay for the visual detection of duck circovirus. Virol J. 2014;11:76.

17. Drew JE, Mayer C-D, Farquharson AJ, Young P, Barrera LN. Custom design of a GeXP multiplexed assay used to assess expression profiles of inflammatory gene targets in normal colon, polyp, and tumor tissue. J Mol Diagn. 2011;13(2):233-42

18. Farquharson A, Steele R, Carey F, Drew J. Novel multiplex method to assess insulin, leptin and adiponectin regulation of inflammatory cytokines associated with colon cancer. Mol Biol Rep. 2012;39(5):5727-36.

19. Rai AJ, Kamath RM, Gerald W, Fleisher M. Analytical validation of the GeXP analyzer and design of a workflow for cancer-biomarker discovery using multiplexed gene-expression profiling. Anal Bioanal Chem. 2009;393(5):1505-11

20. Qin M, Wang DY, Huang F, Nie K, Qu M, Wang M, et al. Detection of pandemic influenza A H1N1 virus by multiplex reverse transcription-PCR with a GeXP analyzer. J Virol Methods. 2010;168(1-2):255-8.

21. Hu X, Zhang $Y$, Zhou $X, X u$ B, Yang $M$, Wang $M$, et al. Simultaneously typing nine serotypes of enteroviruses associated with hand, foot, and mouth disease by a GeXP analyzer-based multiplex reverse transcription-PCR assay. J Clin Microbiol. 2012;50(2):288-93.

22. Yang MJ, Luo L, Nie K, Wang M, Zhang C, Li J, et al. Genotyping of 11 human papillomaviruses by multiplex PCR with a GeXP analyzer. J Med Virol. 2012;84(6):957-63.

23. Xie Z, Luo S, Xie L, Liu J, Pang Y, Deng X, et al. Simultaneous typing of nine avian respiratory pathogens using a novel GeXP analyzer-based multiplex PCR assay. J Virol Methods. 2014;207:188-95.

24. Hu X, Xu B, Yang Y, Liu D, Yang M, Wang J, et al. A high throughput multiplex $P C R$ assay for simultaneous detection of seven aminoglycoside-resistance genes in Enterobacteriaceae. BMC Microbiol. 2013;13:58.

25. Xie Z, Xie L, Fan Q, Pang Y, Deng X, Xie ZQ, et al. A duplex quantitative real-time $P C R$ assay for the detection of Haplosporidium and Perkinsus species in shellfish. Parasitol Res. 2013;112(4):1597-606.

26. Zhang M, Xie Z, Xie L, Deng X, Luo S, Liu J, et al. Simultaneous detection of eight swine reproductive and respiratory pathogens using a novel GeXP analyser-based multiplex PCR assay. J Virol Methods. 2015;224:9-15.

27. Pang Y, Wang H, Girshick T, Xie Z, Khan MI. Development and application of a multiplex polymerase chain reaction for avian respiratory agents. Avian Dis. 2002:46(3):691-9.

28. Xie Z, Pang YS, Liu J, Deng X, Tang X, Sun J, et al. A multiplex RT-PCR for detection of type $A$ influenza virus and differentiation of avian $\mathrm{H} 5, \mathrm{H} 7$, and H9 hemagglutinin subtypes. Mol Cell Probes. 2006;20(3-4):245-9.

29. Huang Q, Yue H, Zhang B, Nie P, Tang C. Development of a real-time quantitative PCR for detecting duck hepatitis a virus genotype C. J Clin Microbiol. 2012;50(10):3318-23.

30. Park SH, Ricke SC. Development of multiplex PCR assay for simultaneous detection of Salmonella genus, Salmonella subspecies I, Salm. Enteritidis, Salm. Heidelberg and Salm. Typhimurium. J Appl Microbiol. 2015;118(1):152-60

31. Zeng Z, Liu Z, Wang W, Tang D, Liang H. Establishment and application of a multiplex PCR for rapid and simultaneous detection of six viruses in swine. J Virol Methods. 2014;208:102-6.

32. Hymas WC, Mills A, Ferguson S, Langer J, She RC, Mahoney W, et al. Development of a multiplex real-time RT-PCR assay for detection of influenza A, influenza B, RSV and typing of the 2009-H1N1 influenza virus. J Virol Methods. 2010;167(2):113-8.

33. Li J, Mao NY, Zhang C, Yang MJ, Wang M, Xu WB, et al. The development of a GeXP-based multiplex reverse transcription-PCR assay for simultaneous detection of sixteen human respiratory virus types/subtypes. BMC Infect Dis. 2012;12:189.

34. Luo SXZ, Xie L, Pang Y, Fan Q, Deng X, Liu J, et al. Development of a GeXP assay for simultaneous differentiation of six chicken respiratory viruses. Bing Du Xue Bao. 2013;29(3):250-7.

35. Zhang YFX, Xie ZX, Xie LJ, Liu JB, Pang YS, Deng XW, et al. Development of a duplex RT-PCR assay for detection of duck tembusu virus and duck plague virus. Journal of Southern Agriculture. 2014;45:314-7. 